\title{
INTERPROFESSIONAL HEALTH EDUCATION TO IMPROVE COLLABORATION IN THE SOUTH AFRICAN CONTEXT: A REALIST REVIEW
}

\section{Carin Maree, Heila van Wyk}

\begin{abstract}
Interprofessional education in health has been identified as a strategy to improve collaborative practice in health care. A need to move away from the training of the various professionals in silos has been identified as a mechanism to address the challenges and changes in the healthcare system. The implementation of interprofessional health education in the health disciplines for undergraduate students in order to promote teamwork among professionals and contribute to improved quality health outcomes is, however, complex and challenging to implement.

The purpose of this article is to identify how, by whom and when interprofessional health education for undergraduate students could be implemented in the South African context by means of a realist review. Interprofessional health education was explored in both developed and developing countries with the focus mainly on developing countries and South Africa in particular. The framework for action on interprofessional education and collaborative practice developed by the World Health Organisation (2010) was used as a reference.

The authors clarified concepts about interprofessional health education, taking into consideration studies about interprofessional education internationally as well as nationally. The intention in this paper is to guide the reader to understand the various measures, context and outcomes associated with the term interprofessional health education'. The measures identified for interprofessional health education involved the following: infrastructure, resources, management, stakeholders' attitudes, teamwork and educational principles. As far as the context is concerned, this differs from developing and developed countries, since the nature of both the health and education systems of these countries differ. Interprofessional health education should take place in a meaningful and relevant context that reflects current or future trends. The desired outcomes for interprofessional health education should include collaboration among health professionals, thus resulting in improved health care.
\end{abstract}

Keywords: Interprofessional health education, collaborative practice, undergraduate students, interdisciplinary education, multi-disciplinary education, transdisciplinary education, multiprofessional education. 


\section{INTRODUCTION}

It is clear that the health system worldwide is faced with major changes and challenges as a result of various contributing factors described by Frenk et al. (2010). These changes and challenges include an increased number of new diseases, as well as lifestyle changes, failure to share knowledge gained to promote healthcare among professionals, poor teamwork, healthcare becoming more costly, and increased workloads for healthcare workers, just to name a few. Healthcare institutions have continued to train professionals in the various disciplines who continue to work in silos in the current healthcare system (Frenk et al., 2010).

Interprofessional education in health care gained significant ground as a mechanism to overcome the challenges in the health system (Frenk et al., 2010) and to enhance collaborative practice, as well as improving health outcomes for patients (World Health Organisation (WHO), 2010). Furthermore, such education is seen as a way of addressing the global shortage of healthcare workers by optimising teamwork among available personnel. Implementation of interprofessional health education in itself poses several challenges as it cuts across different health-related disciplines, contexts with varying demographics and health needs, and a range of educational institutions (WHO, 2010).

Interprofessional health education, which is the primary focus of this article, was identified as one of the strategies to achieve this goal in developing countries such as South Africa. It was assumed that collaboration at undergraduate level would create a basis for collaborative practice in order to prevent potential professionals from learning to practice independently from one another. The question, therefore, was how, by whom and when can interprofessional health education be utilised to improve collaborative practice and health outcomes for patients in developing countries. This was instrumental in the idea of a realist review of the subject.

\section{METHODOLOGY}

Interprofessional health education is complex as it covers different disciplines that have been traditionally presented independently from one another, even if the common goal was to improve health outcomes for patients. It is further complicated by different levels and contexts in which the programmes are presented, with multiple factors of influence. A realist review of the subject was therefore considered in order to expand the knowledge needed for complex interventions by focusing on what works for whom, under what circumstances, as well as how and why, as described by Wong, et al. (2013).

The review was also undertaken to explore aspects of interprofessional health education that 'work', for whom and in what circumstances, and then to unpack possibilities for interprofessional health education in developing countries. The realist review was used to explore the relationship between the mechanism (interprofessional health education of undergraduate students), the context (healthcare in a developing country) and the outcomes (collaboration to improve patient outcomes). For the purpose of the realist review, the WHO's framework for action on interprofessional education and collaborative practice (WHO, 2010) was used as a point of departure, followed by investigating related international and national research. Both quantitative and qualitative articles were included, and the option was kept open to include other relevant sources such as models or descriptions of the progress in interprofessional health education that might contribute to the solution as suggested by Wong et al. (2013). The databases used for an iterative search to find appropriate literature included MEDLINE, PUBMED, CINAHL and Google Scholar. The search was not limited to a particular period of time, but the main focus was on inclusion of literature of the last decade. It was also deemed important to consider a wider scope of investigation to observe the progression of interprofessional health education as it developed or did not develop over time. 

education', 'education for collaborative practice', 'undergraduate health education', 'interdisciplinary education', and 'multi-disciplinary education', 'transdisciplinary education', 'multiprofessional education' and 'interprofessional learning'.

\section{LITERATURE SEARCH}

The selection of relevant literature was conducted first by screening the titles of articles identified through the search. Those initially considered to be potential articles were then screened for suitability, based on the abstracts. These articles were then further screened by reading the full text. The process remained an iterative purposive search as described by Wong et al. (2013).

The basic framework of the WHO (2010) was used as a point of departure for analysis and synthesis. If it appeared that a reference used by the authors in an article might be important, the reference was followed up to search for the particular article which was then also screened for possible inclusion. If the need arose during the review to search for new information for clarification of concepts, more articles were searched for and analysed. The authors collaborated and reached consensus on the concepts to be addressed and the structure in which they should be presented.

The difference between education at undergraduate and postgraduate levels was indicated in the literature. The focus throughout, however, remained on what could work for whom, when and why in terms of interprofessional health education of undergraduate students so as to achieve consensus in terms of improving patient outcomes in the context of a developing country. Postgraduate interprofessional health education was excluded, as it was assumed that health practitioners who were functioning as professionals had already established their methods of practising, whether as part of an interprofessional team, or as individuals. It was further assumed that interprofessional health education for this group would entail different dynamics and would therefore result in different mechanisms for a particular context, as well as different outcomes. The concepts identified were analysed and synthesised to describe the interprofessional health education of undergraduate students (mechanism) in the healthcare of a developing country (context) in order to achieve collaboration for improving patient outcomes (outcomes). Before the findings are discussed, it is necessary to clarify the concepts related to interprofessional health education.

\section{CLARFICATION OF CONCEPTS RELATED TO INTERPROFESSIONAL HEALTH EDUCATION}

Educating healthcare professionals on how to function within a team has emerged as an important aspect of healthcare over the last few decades, but there is no consensus on the use of related terminology. Health education refers to the formal programmes for teaching and learning of health professionals from a variety of disciplines in health care. The associated concepts such as multiprofessional, multidisciplinary, interprofessional, interdisciplinary and transdisciplinary education are often used interchangeably in the literature and can cause confusion among readers. The confusion in the use of terminology is commonly created by conceptual errors (Pirrie, Hamilton \& Wilson, 1999), either related to the prefixes 'inter', 'multi' and 'trans', or to the adjectives 'disciplinary' and 'professional' (Leathard, 1994).

'Inter' implies interdependence that involves team members crossing into another's sphere or surrendering some aspects of their own professional role, altering professional boundaries or accepting a new identity within the team with a form of reprocity between team members (Pirrie et al., 1998; Rawson, 1994). According to Wilmot (1995), 'inter' demands an integrated approach with a greater degree of maturity and flexibility with regard to their knowledge base.

'Multi' implies members working independently, but with related roles, towards the same goal, each team member being responsible for a different part of the patient's treatment with little or no overlapping of professional roles (Pirrie et al., 1998; Wilson \& Pirrie, 2000). Clark (1993) describes 'multi' as 'bringing different perspectives together' in one team. Hammick et al. (2007), on the other hand, are of the opinion that multiprofessional education takes place when 

has an interactive component where they learn with, from and about each other.

The adjectives 'disciplinary' and 'professional' are used interchangeably (Clark, 1993). Pirrie, Hamilton and Wilson (1999) refer to 'multidisciplinary' as the most useful word because it is 'the most ambiguous', and Gilbert, Yan and Hoffman (2010) describe 'professional' as a member who could add skill and knowledge to the well-being of a community. Oandasan and Reeves (2005) reason that although the word 'professional' is commonly used in health care, it could exclude enrolled nursing auxiliaries, massage therapists, and so forth.

'Transdisciplinary education' implies that education transcends the boundarires of two or more disciplines in order to create a holistic approach. The role clarification of the respective disciplines will therefore fade with an increasing overlap of responsibilities (Choi \& Pak, 2006).

The term of choice used in this article is 'interprofessional education', accepting the definitions as stipulated by WHO (2010), namely that "interprofessional education occurs when students from two or more professions learn about, from and with each other to enable effective collaboration and improve health outcomes". For the purpose of this study, sub-categories of healthcare workers such as nursing auxilliaries and other assistants were excluded, with the focus being on undergraduate students in the process of developing as professionals in various healthcare disciplines.

\section{FINDINGS AND DISCUSSION}

The framework for action on interprofessional education and collaborative practice of WHO (2010) was the point of departure for this study; in other words, to find suitable mechanisms related to interprofessional education that would work in the South African context and enhance collaborative practice, as well as improving health outcomes for patients. This was done by means of a realist review, as suggested by Wong et al. (2013).

Examples of qualitative and quantitative studies conducted in interprofessional health education are discussed in the following text, followed by an evaluation of the findings according to the main themes of mechanisms, context and outcomes. The purpose was not to discuss individual studies, but to report on trends related to the themes of interprofessional health education.

\section{Examples of international studies related to interprofessional health education}

The following section provides a brief look at examples of studies conducted in the sphere of interprofessional health education in Australia, Japan and Sweden, as well as North Carolina and Ohio in the United States.

At Monash University in Australia, a study was undertaken where workshops for undergraduate healthcare students formed part of their interprofessional education. During the workshops, a minimum of three healthcare students from different disciplines formed an interprofessional team. The team members could include undergraduate paramedic students, as well as students from nursing, midwifery, occupational therapy, physiotherapy and dietetics. The teams watched DVD simulations of three different conditions, after which they had to work collaboratively to develop optimal healthcare plans for the patients. Six educational components guided these workshops: patient-centred practice; interprofessional learning; communication skills; teamwork and collaboration; conflict management; and reflection. Group activities were added for the purpose of learning about each discipline's professional roles and responsibilities. The authors evaluated the interprofessional education workshop immediately after it had been held and after six months of implementation. The findings indicated that the students' perceptions and attitudes about the interprofessional education workshops were perceived as positive, both directly after the workshop and also after six months. It was concluded that the results could indicate long-term positive outcomes of such interprofessional 

with other disciplines (Williams et al., 2011).

An interprofessional education programme at the University of Gunma in Japan was implemented for the first- and third-year undergraduate healthcare students. Different educational approaches were used for the different year groups. The first-year healthcare students were educated about interprofessional teamwork by means of lectures, including two classes that focused on the details and values of collaborative practice. The third-year students were exposed to a training-style approach with interprofessional teams consisting of four students from nursing, two from speech therapy, one from occupational therapy and one physiotherapy student per group. The interprofessional education training programme consisted of 15 consecutive four-hour lessons and two days of practical fieldwork sessions, followed by a debriefing session and group work to prepare patient reports. The educational approach comprised problem-based learning and collaborative group discussions. Tomoko et al. (2012) then conducted a study to determine the changes in attitudes regarding interprofessional education between the first- and third-year students. The findings indicated that the first-year group's attitudes were inclined to be mainly negative, whereas those of the third-year students were seen as mainly positive. It was concluded that the students' perceptions may have been influenced by the educational approach, since the third-year students experienced a 'meaningful positive change in knowledge and attitude' when exposed to a real-life experience of collaborative practice (Tomoko et al., 2012).

At the University of Columbus in Ohio, a study was conducted to teach students by means of simulation to function within an interprofessional team. These teams consisted of one medical student, one nursing student and a family member per team. They were presented with a standardised scenario where trained members played the role of the patient so that the scenario could be closely related to real life. The team had to work together to devise a plan for optimal patient care. The simulation was found to be 'an effective and a well-received educational intervention' with the potential to foster a culture of interprofessional teamwork (Wagner, Liston \& Miller, 2011).

Since 1986, attempts at interprofessional education have also been implemented by the Linköping University in Sweden. The main aim of the Linköping Interprofessional Education Model was to establish a professional identity for undergraduate healthcare students and to achieve interprofessional competence over a period of time. Medical, nursing, occupational therapy, physiotherapy, biomedical laboratory, medical biology and speech and language undergraduate students were all part of the interprofessional education programme. Problem-based learning in small groups, as well as reflection, formed a fundamental part of the interprofessional education programme. The programme was presented as an eight-week interprofessional education module, with a combination of modules early in the curriculum and practical fieldwork at a student training ward at the end of the programme. The purpose of the training ward was for students to experience how to function within an interprofessional team during a two-week rotation system (Wilhemsson et al., 2009). Staffan et al. (2011) followed up on the study in order to evaluate the effect of the two-week rotation on the undergraduate healthcare students' professional roles and their value in teamwork. The findings indicated improved knowledge of their own professional role, the role of other professions, as well as the benefit of teamwork after exposure to interprofessional teamwork in the training ward.

Rossen, Bartlett and Herrick (2008) used two case studies to describe innovative ways in which the University of North Carolina engaged nursing students in interprofessional collaborative practice other than in hospital settings. The one case study described how interprofessional education for undergraduate nursing students was carried out at a psychiatric unit. Two nursing students were paired for collaboration: one student worked in the morning and the other in the afternoon, but with the same patients. The aim of this activity was to gain skills needed for collaboration such as interpersonal communication and negotiation, which they could then use to participate in interprofessional activities with other team members as well. Strategies to encourage collaboration included time to communicate, plan, evaluate and write down a weekly care plan. Students were also expected to observe interprofessional teamwork from the professionals working in the psychiatric unit. The interprofessional activities were evaluated by the students 

easy task.

A second study by Rossen, Bartlett and Herrick (2008) on interprofessional teamwork was done at a homeless shelter for alcoholic women and their children in North Carolina. Undergraduate students from nursing, psychology, social work, therapeutic creation, human development and special education worked together in a team to provide services to the mothers and their children. Training strategies to promote interprofessional education included formal educational experiences in which the knowledge and skills of the various disciplines were shared. An awareness of the roles of the different members was also created through discussions. Students had to role-play good collaborative practice among team members, as well as discuss their assessment and planning for a specific healthcare user. Rolemodelling by professionals during collaborative practice was another training strategy used to further interprofessional teamwork. The students learned about the similarities and differences of the different team members.

The trends from these international studies on interprofessional health education showed positive results regarding collaborative practice after undergraduate students of interprofessional collaboration underwent meaningful real-life experiences, even if the formats of those real-life experiences differed. It further appeared as if a change in educational approach was the main driving force in facilitating interprofessional health education.

\section{Examples of studies related to interprofessional health education in South Africa}

Treadwell and Havenga (2013) from the University of Limpopo (Medunsa campus) discussed ten key elements when designing and implementing interprofessional learning in clinical simulations - namely, facilitators, learners, patient simulators, content, learning resources, settings, faculty development, logistics, learning strategies and evaluation. The facilitators needed to support new working relationships and to be open to learning and working together. It was suggested that no more than four student groups should be included to create interprofessional teams, as students from multiple disciplines tended to be challenging. Simulation needed to be as close to real life as possible in order to enhance interprofessional learning. Skills needed to work within a team are essential for the educational content including communication, leadership and teamwork. The setting, as well as the details of the logistics, needed to be planned carefully and well in advance. The teaching methods had to be experimental, relevant and meaningful for each professional group. Debriefing, reflection and constant evaluation had to be included in an interprofessional programme (Treadwell \& Havenga, 2013).

Designing a new interprofessional education curriculum proved to be a challenge. Three articles that describe the process of developing such an interprofessional education curriculum in the South African context have already been published. The aim of these articles was to assist other higher educational institutions in the process of developing interprofessional curricula (Waggie \& Laattoe, 2014).

Waggie and Laattoe (2014) described the process of developing an interprofessional programme designed for all undergraduate students in nursing, physiotherapy, occupational therapy, social work, dietetics, natural medicine, and sport science and recreation at the University of the Western Cape in South Africa. Three exemplars guided the development of this programme: community-based service learning with an underlying pedagogical approach, interprofessional community-based practice, and communication in interprofessional teamwork. Teaching and learning methods included small group discussions, didactic input, video clips, role-play, case studies, presentations, and working within an interprofessional team, while recognising the different members' roles and responsibilities. Some of the lessons learnt by the authors were that support from the university and faculties proved to be essential, that the interprofessional education committee should be represented by all the faculties involved and that the coordination and logistics of bringing several disciplines together are challenging (Waggie \& Laattoe, 2014). 

University of Cape Town (Duncan et al. 2006; Mayers et al. 2006). The first article described the reasons why the curriculum was transformed and the second the practicalities of the curriculum design process. A shift from more traditional multiprofessional educational courses occurred in order to unite rather than differentiate among the professions. The teaching methods planned were small group participation in experiential and community projectbased learning. The course was designed to focus on the following objectives: development of interpersonal relationships, understanding group dynamics, professionalism, commitment to human rights and endorsement of the primary healthcare philosophy. The entire group of first-year undergraduate students in health professions at the Faculty of Health Science at the University of Cape Town were included in this course. The basis of the curriculum content was to focus on a commitment to sound professional relationships with colleagues, clients and the public, as well as commitment to primary health care. The main aim of designing a new curriculum was that students should work more collaboratively, develop knowledge, attitudes and skills that would assist them to treat a patient in a holistic manner and ultimately improve patient outcomes. The authors indicated that designing a new multiprofessional education curriculum was not always an easy task as it required educators to abandon certain preconceived ideas, take time to plan, reach consensus (which was often accompanied by conflict), communicate with one another and ensure support structures. Other challenges that the design team had to face were the difficulty to coordinate the new course in the timetable, time pressures, scepticism and resistance to changing the current way of doing things. Despite the challenges of designing a new curriculum, the members of the design team succeeded in developing an understanding of each other's roles, responsibilities, strengths and contributions (Duncan et al., 2006; Mayers et al., 2006).

In the above examples, it is clear that interprofessional education can take on different forms in different settings and follow different approaches, but that the aim remains the same - focusing on improving collaborative practice. Meaningful real-life experiences appear to be a central theme. Various mechanisms related to interprofessional health education were indicated in the different contexts, with the main outcome being collaborative practice. These aspects are discussed in the following section.

\section{Mechanisms of interprofessional health education}

The concept 'mechanisms' refers to the underlying entities, processes or structures (including social structures) which operate in specific contexts to generate outcomes of interest (Wong et al., 2013). In this case, mechanisms are related to interprofessional health education as an intervention, and the trends identified are therefore described with a focus on the underlying who, what and how of interprofessional health education.

\section{Health professions involved in interprofessional health education}

The different health professions indicate 'who is involved' in interprofessional health education. The Study Group on Interprofessional Education and Collaborative Practice of the WHO conducted a study in 2008 to determine the global status of interprofessional education programmes in 42 countries. The respondents $(n=396)$ represented education $(50,4 \%)$, practice $(14,1 \%)$, research $(11,6 \%)$ and administration $(10,6 \%)$. The learners involved in interprofessional education programmes included nurses/midwives $(16 \%)$, doctors/physicians $(10,2 \%)$, physiotherapists $(10,1 \%)$, social workers $(9,3 \%)$, occupational therapists $(8,9 \%)$, pharmacists $(7,7 \%)$, psychologists $(5,9 \%)$, nutritionists / dieticians $(5,7 \%)$, speech pathologists $(4,7 \%)$, community health workers $(4,3 \%)$, audiologists $(2,2 \%)$, physicians' assistants $(2,2 \%)$ and podiatrists $(1,6 \%)$. The remaining $6,7 \%$ included all other types of health workers not involved in interprofessional health education programmes (WHO, 2010).

Cooper et al. (2001) found the following professions to be involved in interprofessional education: nursing, medicine, social work, pharmacology, dentistry, laboratory science, speech therapy, dietetics, audiology, occupational therapy, 

professions that participate most often in interprofessional education: medicine, nursing and midwifery, physiotherapy, pharmacology, occupational therapy, dentistry and social work.

A common theme in the literature was that interprofessional education should not replace profession-specific education, but should contribute to a depth of education in which the students from the different professions learn from, with and about each other, and with learning domains aligned with generic aspects of health care and collaborative practice (Cooper et al., 2001; Hammick, 2007; WHO, 2010).

It appears that the only criterion for health professions to be successfully involved in interprofessional education is to have a common goal of improving health outcomes for patients and communities, and in particular not the role of the respective profession in health care.

\section{Infrastructure, resources and management}

For interprofessional education to be successful, important mechanisms that need to be in place include supportive institutional and management practices, potential champions to drive the process, and a resolve to change the culture and attitudes of health workers. If necessary, health systems need to be reviewed in order to create an environment in which healthcare workers can implement collaborative practices (WHO 2010). This is relevant for both educational and healthcare institutions.

Treadwell and Havenga (2013), Frenk et al. (2013), Bridges et al. (2011), Duncan et al. (2006), Mayers et al. (2006) and Cooper et al. (2001) all support the WHO's (2010) recommendations. They emphasise the importance of the availability of infrastructure and resources from faculties, departments and staff, and well-managed planning and community relationships which are essential for implementing interprofessional education and collaborative practice.

Interventions regarding interprofessional education require detailed and committed team planning and increased resources (Cooper et al., 2001; Treadwell \& Havenga, 2013). Resources that are critical for a successful interprofessional education programme include adequate physical space, technology, administrative support and interprofessional programmatic infrastructure (Bridges et al., 2011; Treadwell \& Havenga, 2013; WHO, 2010).

Committed and appropriate infrastructure, resources and management are identified as crucial prerequisites for the implementation of interprofessional health education in order to improve collaborative practice.

\section{Stakeholders' attitudes and teamwork}

Further essential mechanisms for the successful implementation of interprofessional education include commitment from faculties, departments and staff of educational as well as healthcare institutions. A shared vision and understanding of the benefits of introducing a new interprofessional education curriculum is vital. Other important mechanisms include enthusiasm for the work being done, training of staff for interprofessional education and collaboration, good communication among participants, and a willingness to update information, renew and revise existing institute-appropriate legislation to eliminate barriers to collaborative practice by addressing health systems (WHO, 2010).

Mayers et al. (2006) emphasised the importance of finding a common vision and planning structure, agreement on the principles to guide course design, and acknowledgement of the strengths and roles of team members. The stakeholders should 'buy into' the concept. 

for its effectiveness. Personel need to understand the principles of interprofessional education and the multiple factors that influence learning (Hammick et al., 2007). Bridges et al. (2011) emphasised the importance of mentoring and faculty training. Treadwell and Havenga (2013) added that committed facilitators who encourage collegial learning, change thinking and support new working relationships are needed for successful interprofessional education programmes (Treadwell \& Havenga, 2013).

Among the stakeholders, the importance of teamwork in the planning and preparation for interprofessional health education was identified as a significant requirement, as well as an attitude of commitment, support and willingness to introduce or accept change.

\section{Education principles}

The WHO (2010), Cooper et al. (2001) and Hammick et al. (2007) recommended that the principles of adult learning should be followed during interprofessional education programmes. Bridges et al. (2011) agreed, and emphasised the importance of appropriate curricular mapping to make interprofessional education relevant. Treadwell and Havenga (2013) indicated that the content of the programme should be based on shared objectives, while Hammick et al. (2007) emphasised the importance for interprofessional education to take place in a context that reflects the students' current or future practices.

The WHO (2010) argued that team members first needed to establish a discipline-specific foundation of knowledge and skills before they could function optimally within a team, an opinion supported by Mariano (1989), Clark (1993), Cooper et al. (2001), and Hammick et al. (2007). Burch (2014) continued with the argument that specialised knowledge and skills are crucial in the respective professions, but also that the foundation for collaborative practice and teamwork among the professions should be established as early as possible so as to prevent stereotyping and professional arrogance and to create mutual respect and understanding.

Burch (2014) maintained that the curricula for undergraduate health profession programmes should include interprofessional learning early on. This was supported by Williams et al. (2011), Wagner et al. (2011), Staffan et al. (2011), Rossen et al. (2008), Duncan et al. (2006), Waggie and Laattoe (2014) and Treadwell and Havenga (2013). Cooper et al. (2001) maintained that early learning experiences were most beneficial in developing healthy attitudes toward interprofessional teamwork before stereotyping became strongly formed.

The WHO (2010) listed the following main learning domains for interprofessional education:

- Teamwork:

- Being able to be both team leader and team member;

- Knowing the barriers to teamwork.

- Roles and responsibilities:

- Understanding one's own roles, responsibilities and expertise;

- Understanding those of other types of healthcare workers.

- Communication:

- Expressing one's opinions competently to colleagues;

- Listening to team members.

- Learning and critical reflection:

- Reflecting critically on one's own relationship within a team.

- Transferring interprofessional learning to the work setting:

○ Relationship with, and recognising the needs of, the patient; 
○ Working collaboratively in the best interest of the patient;

- Engaging with patients, their families, carers and communities as partners in care management.

- Ethical practice relevant to the profession:

- Understanding the stereotypical views of other healthcare workers held both by oneself and by others;

- Acknowledging that each healthcare worker's views are equally valid and important.

Additional content related to skills required for teamwork includes leadership (Treadwell \& Havenga, 2013), conflict management, prioritising and decision-making (Williams et al. 2011), reflection and practice-related content, e.g. primary healthcare, screening for risk factors, etc. (Hammick et al. 2007). Burch (2014) further argued that the curricula of undergraduate health profession programmes should include interprofessional learning, with an emphasis on the central values of professionalism, namely, altruism, accountability, excellence, duty, advocacy, service, honour, integrity, respect for others as well as ethical and moral standards and the acquisition of skills needed to function as part of a multiprofessional team. The focus of the content should mainly be on the underlying principles of interprofessional collaboration, which can be applied in different settings. Health challenges in which interprofessional education and collaborative practice can make a positive contribution include family and community health, HIV/AIDS, tuberculosis, malaria, humanitarian crises and conflicts, health security threatened by epidemics and pandemics, noncommunicable diseases, mental health, human resource shortage in health systems and healthcare services (WHO, 2010).

\section{Suggested teaching strategies in interprofessional health education}

A wide variety of teaching strategies is recommended. Cooper et al. (2001) identified problem-based learning, small group teaching, case studies and experiential work as approaches that contribute to the success of interprofessional health education. Treadwell and Havenga (2013) added the inclusion of a range of assessment methods, including student surveys, as contributing to efficient interprofessional education. Teaching, learning and assessment should be authentic and should therefore take place in a meaningful and relevant context that either is, or reflects, real-world practices and includes active participation, experiential learning, debriefing and reflection (Treadwell \& Havenga 2013; Hammick et al., 2007). This is also supported by the examples of studies discussed in the previous section.

Outstanding trends regarding the underlying education principles of interprofessional health education stress the importance of such principles to the students' current and/or future practices, and in reflecting real-life situations. The content should be focused on various aspects of teamwork and generic aspects of health care, and not disciplinespecific aspects. A wide variety of teaching, learning and assessment strategies are recommended, with experiential learning, debriefing and reflection considered as being important.

\section{Interprofessional health education context}

The context refers to where and when the phenomenon under study takes place (Wong et al., 2013). To explore the context of interprofessional health education, the current situation of health systems, as well as education systems in developed and developing countries should be considered. The need for a different cadre of healthcare professionals originated in changes in health systems worldwide, which required education institutions to introduce changes in health education. Because of these changes, interprofessional health education emerged as an essential strategy in health education in both developed and developing countries (WHO, 2010; Frenk et al., 2010).

Challenges that persist in both developing and developed countries include an increase in the complexity and cost of health systems, static health education curriculums, professionals in the various fields functioning in silos, dysfunctional health systems and an inability to adapt to the context (Frenk et al., 2010). 

expectancy of the people over the last decade in developed countries, and a lowered life expectancy in developing countries. The increase in life expectancy might be due to improved life standards and increased healthcare knowledge. The opposite in the developing countries could be associated with the HIV/AIDS pandemic and poverty that continues to play a major role in morbidity and mortality (Frenk et al., 2010). The situation is aggravated by a lack of resources and healthcare staff shortages in developing countries (Frenk et al., 2010; WHO, 2010). The attention in both developed and developing countries tends to focus on the implementation of urgent action plans rather than on assuring improved, flexible and updated health education related to the changing health system (Frenk et al., 2010).

This is also relevant for South Africa as a developing country. Characteristics of the South African health context necessitated revising education for healthcare professionals. The health system is divided into public and private health sectors, with vast differences in terms of quality and access. There is a quadruple burden of disease, namely a very high prevalence of HIV and AIDS (especially in association with tuberculosis); maternal and child morbidity and mortality; the exploding prevalence of non-communicable diseases; and violence, injuries and trauma. Furthermore, there is a shift to the re-engineering of primary health care, the introduction of the National Health Insurance System and several other strategies to address the issues mentioned (e.g. HIV Counselling and Testing; Nurse-Initiated Management of the Antiretroviral Therapy Programme (NIMART), and Prevention of Mother-to-Child Transmission (PMTCT)) (Department of Health, RSA ,2014; Benatar, 2013).

According to Benatar (2013), factors which aggravate the challenges experienced in health care in South Africa include the widespread economic crisis and increasing poverty, where people in need of health care do not have money for transport to healthcare facilities, or are unable to comply with special dietary needs, as well as attendant fraud and corruption. Further problems include a lack of resources and infrastructure in rural areas (Mpofu et al., 2014). A shortage of healthcare providers is a global trend and is a serious concern in South Africa as it affects the rural areas in particular (WHO, 2013; Africa Health Placements, 2015). The shortages are expected to become even worse, due to the ageing health force, poor salaries and a lack of incentives to keep healthcare professionals in their respective professions (WHO, 2013). The effect of staff shortages on the quality of patient care is further exacerbated by the lack of collaboration between different disciplines (professionals working in silos), both in the private and public sectors (Parrish \& Blockman, 2008).

Treadwell and Havenga (2013), Hammick et al. (2007) and WHO (2010) all described teaching, learning and assessment as authentic and felt that it should therefore take place in a meaningful and relevant context that is either consistent with, or reflects, real world practices. Cameron, Rutherford and Mountain (2012) maintained that interprofessional education should be embedded in work-based learning in order to promote collaborative practice. For effective learning to be achieved, interprofessional teamwork is essential in a context that reflects the students' current or future practice (Hammick et al., 2007).

It can therefore be concluded that there are multiple challenges in health systems worldwide, whether in developed or developing countries, or in the private or public sectors. These challenges demand a different generation of healthcare professionals to be trained to cope with these obstacles while delivering quality health care and improving health outcomes of patients. Interprofessional health education, where the focus is on the development of healthcare professionals for collaborative healthcare practice, emerged as a strategy to address these demands. An important factor in the development of professionals to learn with and from each other has been identified. Furthermore, the context in which interprofessional education takes place should be authentic and relevant and needs to reflect the real world to which students will be exposed as healthcare professionals.

\section{Outcomes of interprofessional health education}



(2010) described interprofessional education as an innovative step to enable collaboration and improve health outcomes in terms of local health needs. Collaborative practice occurs when healthcare workers from different professional backgrounds provide clinical and non-clinical comprehensive services by working with other professionals, with patients, their families, carers and communities to deliver the highest quality of care across various spheres (WHO, 2010). Interprofessional education leading to collaborative practice is seen as one of the advanced strategies to address the call for scaling up health workforce production to ensure an appropriate supply, mix and distribution of the health work force (WHO, 2010). Improved collaboration, better-quality health outcomes and a stronger workforce can therefore be seen as important outcomes of interprofessional education which are also relevant and needed in the South African context.

The outcomes further affect two key stakeholders - namely, the patients receiving the service and the healthcare providers responsible for delivering the service (WHO, 2010). Improved health outcomes are expected when professionals from different disciplines work together and share responsibilities to achieve optimal health outcomes for patients. Patient satisfaction is seen as an important outcome for the patients receiving the service, which is expected when they receive holistic care. For healthcare providers, the end result of interprofessional education would be collaborative health workers ready for practice. The healthcare providers need to work together to achieve local health goals, move away from non-collaboration or fragmentation in health care and build stronger health systems, which in turn improve health outcomes for patients. According to the WHO (2010), additional outcomes for healthcare providers would include sharing knowledge of the best practices pertaining to work-force satisfaction and well-being.

Internationally, the result of global changes in healthcare needs, specifically as these relate to an ageing population, together with an increase in chronic diseases and accompanying disability in developed countries, morbidity associated with poverty and infective diseases such as HIV/AIDS in developing countries, have caused changes to healthcare provision. There is, therefore, also a need to change the education of healthcare professionals (Burch, 2014; Cooper et al., 2001; Phillippon et al., 2005).

Burch (2014) indicated that the focus should be shifted from curing to the controlling of symptoms, optimising quality of life and coping with long-term conditions which require a higher level of collaboration between them. This in turn calls for better teamwork for which healthcare professionals should be prepared for during their undergraduate studies.

Cooper et al. (2001) explained that the effects of interprofessional education are related to improved changes in knowledge, skills, attitudes and beliefs. Phillippon et al. (2005) concurred, and stated that the changes as a result of interprofessional education were contributing to more effective work and collaboration in interdisciplinary teams among health professionals and, in the end, benefited both patients and communities.

A new shift to implement a systems approach was suggested by the Commission in Health Education as described by Frenk et al. (2010). This includes improving health systems by adapting core professionals' competencies to specific contexts, while making use of global knowledge. A key element in the systems approach is interdependence, with the focus on collaborative education and networking with other institutions. This entails sharing of educational content, teaching resources and innovative practices (Frenk et al., 2010).

Pumar Mendez et al. (2008) indicate that although the need for interprofessional education is supported by evidence, it does not go without obstacles and challenges. Some of the challenges include lack of time, scarce financial resources, varying educational schedules and discipline-specific requirements for registration (Cooper et al., 2001). Hammick et al. (2007) also add stereotyping, unwillingness to cooperate and incompetent educators as obstacles. Despite the challenges, mutual learning, interdependence in health and shared education have evolved and have expanded over the last decade (Frenk et al., 2010). 


\section{TABLE 1: SUMMARY OF THE MECHANISMS, CONTEXT AND OUTCOMES OF INTERPROFESSIONAL} EDUCATION

MECHANISMS (who, what and how?)

Different health professionals are involved in interprofessional education

- Nurses, doctors, physiotherapists, social workers, occupational therapists, pharmacists, psychologists, dieticians, speech pathologists, community health workers, audiologists, physicians' assistants, podiatrists, health administrators, dentists etc. (WHO, 2010; Cooper et al., 2001; Hammick et al., 2007).

\section{Infrastructure, resources and management}

- Institutional and management practices should be supportive (WHO, 2010)

- Members are needed to drive the process to facilitate collaboration (WHO, 2010)

- Health systems should be reviewed (WHO, 2010)

- Have suitable infrastructure and resources to facilitate collaboration (Treadwell \& Havenga, 2013; Frenk et al., 2013; Bridges et al., 2011; Duncan et al., 2006; Mayers et al., 2006; Cooper et al., 2001)

- Community relationships should be established (Treadwell \& Havenga, 2013; Frenk et al., 2013; Bridges et al., 2011; Duncan et al., 2006; Mayers et al., 2006; Cooper et al., 2001)

- Detailed and committed team planning should be done (Cooper et al., 2001; Treadwell \& Havenga, 2013)

- Increased resources are needed (Cooper et al., 2001, Treadwell \& Havenga, 2013)

- Adequate physical space, technology, administrative support is essential (Bridges et al. 2011; Treadwell \& Havenga, 2013; WHO, 2010)

- Interprofessional programmatic infrastructure is needed (Bridges et al., 2011; Treadwell \& Havenga, 2013; WHO, 2010)

\section{Stakeholders' attitude and teamwork}

- Stakeholders should have a shared vision of the benefit of collaboration (WHO, 2010)

- The staff should be trained for interprofessional education and collaboration (WHO, 2010)

- Good communication between stakeholders is essential (WHO, 2010)

- There should be a willingness to update information (WHO, 2010)

- Stakeholders should 'buy into' the concept of collaboration (Mayers et al., 2006)

- Staff development and mentoring should take place (Hammick et al., 2007; Bridges et al., 2011)

- New working relationships should encourage mutual support(Treadwell \& Havenga, 2013)

\section{Education principles}

- It is recommended to use the adult learning theory (WHO, 2010; Cooper et al., 2001 and Hammick et al., 2007)

- The content of interprofessional education should be based on shared objectives (Treadwell \& Havenga, 2013)

- Discipline-specific foundation of knowledge should be established first (WHO, 2010; Mariano, 1989; Clark, 1993; Cooper et al., 2001; Hammick et al., 2007)

- Establish specialised knowledge and skill and the foundation for collaboration (Burch, 2014)

- Prevent stereotyping and professional arrogance (Burch, 2014)

- Create mutual respect and understanding (Burch, 2014)

- $\quad$ Suggested learning domains (WHO, 2010)

○ Teamwork

- Roles and responsibilities

- Communication

- Learning and critical reflection

- Transferring interprofessional learning to the work setting

- Ethical practice:

- Additional skills: leadership (Treadwell \& Havenga, 2013), conflict management, prioritising and decision-making (Williams et al., 2011), reflection and practice-related content (Hammick et al., 2007), central values of professionalism, namely altruism, accountability, excellence, duty to care, advocacy, 
service, honour, integrity, respect for others, as well as ethical and moral standards, and the

acquisition of skills needed to function as part of a multi-professional team(Burch, 2014)

- Variety of teaching strategies such as problem-based learning, small group teaching, case studies and experiential work could be used (Cooper et al., 2001)

- Teaching, learning and assessment should be authentic and should therefore take place in a meaningful and relevant context (Treadwell \& Havenga, 2013, Hammick et al., 2007)

\section{CONTEXT (where and when?)}

- Context for interprofessional education differs in developed and developing countries as the health situation in the different countries also differs (Frenk et al., 2010)

- In developed countries the life expectancies increased due to improved standards of living and increased healthcare knowledge (Frenk et al., 2010)

- In developing countries the life expectancies decreased due to the HIV/AIDS pandemic and poverty (Frenk et al., 2010)

- Interprofessional education should take place in a meaningful, relevant context that reflects current or future settings for team members (Hammick et al., 2007)

\section{OUTCOMES (why?)}

- Interprofessional education improves collaboration and health outcomes (WHO, 2010;Phillippon et al. 2005)

- Interprofessional education aims at strengthening the work force (WHO, 2010)

- Collaborative practice has the potential to improve patient satisfaction (WHO, 2010)

- Interprofessional education has, according to Cooper et al (2001), change knowledge, skills, attitudes and beliefs for the better

- Interdependence amongst professions and mutual learning are preferred outcomes of interprofessional education (Frenk et al., 2010)

\section{RECOMMENDATIONS}

It is recommended that formal interprofessional health education programmes should be developed and implemented, and that the impact on the health and education systems, on patients, communities and healthcare providers should be monitored.

\section{CONCLUSION}

Interprofessional health education is frequently used as a mechanism for enhancing the development and improvement of the practice and quality of healthcare services in developed and developing countries. Its main purpose is to enhance collaboration between and amongst undergraduate health professionals. Various authors support interprofessional education as important for establishing a culture of collaboration and teamwork among different healthcare professions at an early stage of professional development in order to achieve improved health care outcomes for patients and healthcare providers alike. Any health care discipline that serves the same patients and communities and has a common goal to improve the health outcomes for these patients and communities can be included.

The context of interprofessional healthcare education worldwide is related to countries' health systems as a driving force to demand collaborative healthcare professionals. These demands then impact on the education systems to develop healthcare professionals who are able to work together with a common goal of improved practice, and to learn from and with each other. The education to develop such professionals does not replace discipline-specific knowledge and skills, but it removes the use of the knowledge and skills in silos by sharing what is common. Common themes to be addressed in interprofessional health education include teamwork, roles and responsibilities of different disciplines, communication, reflection, patient- and family-centred care, ethical practice, leadership and the central 

principles of adult education and to be authentic in reflecting real-life healthcare practice.

In spite of several challenges, interprofessional health education can contribute to collaboration and teamwork, which in turn can lead to better health outcomes for patients and healthcare workers in a country such as South Africa.

\section{ACKNOWLEDGEMENTS}

The following persons are acknowledged: Dr Marianne de Beer for her contribution as critical reader and Wessel Germishuys for his contribution in editing the article.

\section{REFERENCES}

Africa Health Placements,. 2015. The need: no people = no healthcare, Online: http://www.ahp.org.za/no-people-nohealthcare.

Benatar, S. 2013. The challenges of health disparities in South Africa (Editorial). The South African Medical Journal 103 (3): 154-155. Online DOI:10.7196/SAMJ.6622.

Bridges, D.R., Davidson, R.A., Odegard, P.S., Maki I.V. \& Tomkowiak, J. 2011. Interprofessional collaboration: three best practice models of interprofessional education. Medical Education Online 16(10): 3402/meo.v16i0.6035. PMCID: PMC3081249.

Burch, V. 2014. Editorial: Interprofessional education - is it 'chakalaka' medicine? African Journal of Health Professions Education 6 (1): 2.DOI:10.7196/AJHPE.

Cameron, S., Rutherford, I. \& Mountain, K. 2012. Debating the use of work-based learning and interprofessional education in promoting collaborative practice in primary care: a discussion paper. Quality in Primary Care 20 (3): 211 217. PMID: 22828676 [Pubmed - indexed for MEDLINE].

Clark, P.G. 1993. A typology of multidisciplinary education in gerontology and geriatrics: are we really doing what we say we are? Journal of Interprofessional Care 7 (3): 217-228.

Choi, B.C. \& Pak, A.W. 2006. Centre for Chronic Disease Prevention and Control, Public Health Agency of Canada, Ottawa, Ontario. Clinical and Investigative Medicine. Medecine Clinique et Experimentale 29 (6): $351-364$.

Cooper, H., Carlisle, C., Gibbs, T. \& Watkins, C. 2001. Developing an evidence base for interdisciplinary learning: a systematic review. Journal of Advanced Nursing 35 (2): 228-237.

Department of Health, Republic of South Africa, 2014.Strategic Plan: 2014/15 - 2018/19. Viewed 8 April 2015 from http://www.health-e.org.za/wp-content/uploads/2014/08/SA-DoH-Strategic-Plan-2014-2019.pdf

Duncan, M., Alperstein, M., Mayers, P., Olckers, L., \& Gibbs, T. 2006. Not just another multi-professional course! Part 1. Rationale for a transformative curriculum. Medical Teacher 28 (1): 59-63. 

century: transforming education to strengthen health systems in an interdependent world. The Lancet 376 (9756): 1923-1958. Viewed 31 March 2015 from http://www.thelancet.com

Gilbert, J. H., Yan, J. \& Hoffman, J. 2010. A WHO report: Framework for action on interprofessional education and collaborative practice. Journal of Allied Health 39 (1): 196-197.

Hammick, M., Freeth, D., Koppel, I., Reeves, S., \& Barr, H. 2007. A best evidence systematic review of interprofessional education: BEME Guide no. 9. Medical Teacher 29 (8): 735-51.

Leathard, A. 1994. Going Interprofessional, Working Together for Health and Welfare. London: Routledge.

Mayers, P., Alperstein, M., Duncan, M., Olckers, L., \& Gibbs, T. 2006. Not just another multi-professional course! Part 2: Nuts and bolts of designing a transformed curriculum for multi-professional learning. Medical Teacher 28 (2): $152-$ 157.

Mariano, C. 1989. The case for interdisciplinary collaboration. Nursing Outlook 37 (6): 285.

Mpofu, R, Daniels, P.S., Adonis, T. \& Karaguti, W.M. 2014. Impact of an interprofessional education program on developing skilled graduates well-equipped to practise in rural and underserved areas. Rural and Remote Health 14: 2671. Online: http://www.rrh.org.au

Oandasan, I., \& Reeves, S. 2005. Key elements for interprofessional education, Part 1: The learner, the educator and the learning context. Journal of Interprofessional Care 19 (1): 21-38.

Parrish, A. \& Blockman, M. 2008. Clinical excellence and the NICEties of value-based priority setting. South African Medical Journal $98 \quad$ (10): Online: $\quad$ http://www.scielo.org.za/scielo.php?pid=S0256$\underline{95742008001000009 \& \text { script }=\text { sci arttext }}$

Pirrie, A., Wilson, V., Elsegood, J., Hall, J., Hamilton, S., Harden, R., Lee, D. \& Stead. 1998. Evaluating multidisciplinary education in healthcare. Edinburgh: Scottish Council for Research in Education.

Pirrie, A., Hamilton, S. \& Wilson, V. 1999. Multidisciplinary education: some issues and concerns. Educational Research 41 (3): 301-14.

Pumar Méndez, M.J., Armayor, N.C., Díaz Navarlaz, M.T. \& Wakefield, A. 2008. The potential advantages and disadvantages of introducing interprofessional education into the healthcare curricula in Spain. Nurse Education Today 28 (3): 327-36.

Rawson, D. 1994. Models of interprofessional work. Likely theories and Possibilities.

Rossen, E.K., Bartlett, R. \& Herrick, C.A. 2008. Interdisciplinary collaboration: The need to revisit. Issues in Mental Health Nursing 29 (4): 387-96.

Staffan, P., Kalen, A., Hammar, M. \& Wahlström, O. 2011. Preparation for becoming members of healthcare teams: findings from a 5-year evaluation of a student interprofessional training ward. Journal of Interprofessional Care 25 (5): 328-332.

Tomoko, H., Shinozaki, H., Makino, T. Ogawara, H., Yasuyoshi, A., Iwasaki, K., Matsuda, T., Abe, Y., Tozato, F., Koizumi, M., Yasukawa, A., Lee, B., Hayashi, K. \& Watanabe, H. 2012. Changes in attitudes toward interprofessional healthcare teams and education in the first-and third-year undergraduate students. Journal of Interprofessional Care 26 (2): 100107. 

simulations. African Journal of Health Professions Education 5 (2): 80-83. DOI:10.7196/AJHPE.233.

Waggie, F. \& Laattoe, N. 2014. Interprofessional exemplars for health professional programmes at a South African university. Journal of Interprofessional Care 28 (4): 368-370. DOI:10.3109/13561820.2014.891572, Epub 2014 Feb 25.

Wagner, J., Liston, B., \& Miller J. 2011. Developing interprofessional communication skills. Teaching and Learning in Nursing Skills 6 (3): 97-101.

WHO Health Professions Network Nursing and Midwifery Office, Department of Human Resources for Health. 2010. Framework for action on interprofessional education and collaborative practice. http://www.who.int/hrh/nursing midwifery/en/

WHO, 2013, A universal truth: no health without a workforce, Online: http://who.int/entity/workforcealliance/knowledge/resources/hrhreport2013/en/-22k

Wilhelmsson, M., W., Pelling, S., Ludvigsson, J., Hammar, M., Dahlgren, L. \& Faresjö, T. 2009. Twenty years experiences of interprofessional education in Linköping - ground-breaking and sustainable. Journal of Interprofessional Care 23 (2): 121-133.

Williams, B., Brown, T., McCook F., Boyle M., Palermo C., Molloy A., McKenna L., Scholes, R, French, J. \& McCall L. 2011 A pilot study evaluating an interprofessional education workshop for undergraduate healthcare students. Journal of Interprofessional Care 25 (3): 215-7.

Wilmot, S. 1995. Professional values and interprofessional dialogue. Journal of Interprofessional Care 9 (3): $257-65$.

Wilson, V., Pirrie, A. 2000. Multidisciplinary teamworking: beyond the barriers?: A review of the issues. Edinburgh: SCRE.

Wong, G., Greenhalgh, T., Westhorp, G., Buckingham, J., Pawson, R. 2013. RAMESES publication standards: realist syntheses. BMC Medicine http://www.biomedcentral.com/1741-7015/11/21 